\title{
Synthetic Studies on the Taxane Skeleton: Construction of Eight-membered Carbocyclic Rings by the Intramolecular B-Alkyl Suzuki-Miyaura Cross-coupling Reaction
}

Hatsuo Kawada, Mitsuhiro Iwamoto, Masayuki Utsugi, Masayuki Miyano, and

Masahisa Nakada*

Department of Chemistry, School of Science and Engineering, Waseda University

3-4-1 Ohkubo, Shinjuku-ku, Tokyo 169-8555, Japan

General Information. ${ }^{1} \mathrm{H}$ and ${ }^{13} \mathrm{C}$ NMR spectra were recorded on a JEOL AL-400 and AL-300 spectrometer. ${ }^{1} \mathrm{H}$ and ${ }^{13} \mathrm{C}$ chemical shifts are reported in ppm downfield from tetramethylsilane (TMS, $\delta$ scale) with the solvent resonances as internal standards. The following abbreviations were used to explain the multiplicities: s, singlet; $d$, doublet; t, triplet; q, quartet; m, multiplet; band, several overlapping signals; br, broad. IR spectra were recorded on a JASCO FT/IR-8300. Melting points (mp) are uncorrected, recorded on a Yamato capillary melting point apparatus. Optical rotations were measured using a $2 \mathrm{ml}$ cell with a $1 \mathrm{dm}$ path length on a JASCO DIP-1000. Mass spectra and elemental analyses were provided at the Materials Characterization Central Laboratory, Waseda University. All reactions were carried out under an argon atmosphere with dry, freshly distilled solvents under anhydrous conditions, unless otherwise noted. All reactions were monitored by thin-layer chromatography carried out on $0.25 \mathrm{~mm}$ E. Merck silica gel plates (60F-254) using UV light as visualizing agent and phosphomolybdic acid and heat as developing agents. E. Merck silica gel (60, particle size 0.040-0.063 mm) was used for flash column chromatography. Preparative thin-layer chromatography (PTLC) separations were carried out on self-made $0.3 \mathrm{~mm}$ E. Merck silica gel plates (60F-254).

Materials. THF was distilled from sodium/benzophenone ketyl, and methylene chloride $\left(\mathrm{CH}_{2} \mathrm{Cl}_{2}\right)$, and acetonitrile from calcium hydride. Toluene was distilled from sodium. DMF was distilled from $\mathrm{CaH}_{2}$ under reduced pressure.

\section{1-(2-Iodo-phenyl)-hex-5-en-1-ol (a)}

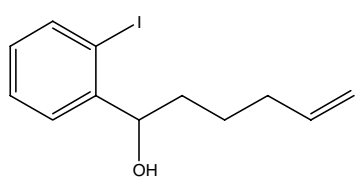

IR (thin film) $v_{\max }: 3356,2940,1640,1462,1436,1010,914,754 \mathrm{~cm}^{-1} ;{ }^{1} \mathrm{H}$ NMR $\left(400 \mathrm{MHz}, \mathrm{CDCl}_{3}\right)$ : $\delta 7.79(\mathrm{~d}, J=7.3 \mathrm{~Hz}, 1 \mathrm{H}), 7.51(\mathrm{~d}, J=7.3 \mathrm{~Hz}, 1 \mathrm{H}), 7.37(\mathrm{dd}, J=7.3,7.3 \mathrm{~Hz}, 1 \mathrm{H}), 6.97(\mathrm{dd}, J=7.3,7.3 \mathrm{~Hz}$, 
1H), 5.87-5.77 (m, 1H), $5.03(\mathrm{~d}, J=17.1 \mathrm{~Hz}, 1 \mathrm{H}), 4.96(\mathrm{~d}, J=10.3 \mathrm{~Hz}, 1 \mathrm{H}), 4.92-4.90(\mathrm{~m}, 1 \mathrm{H}), 2.17-2.08$ $(\mathrm{m}, 2 \mathrm{H}), 19.4-1.93(\mathrm{~m}, 1 \mathrm{H}), 1.79-1.69(\mathrm{~m}, 1 \mathrm{H}), 1.67-1.61(\mathrm{~m}, 2 \mathrm{H}) ;{ }^{13} \mathrm{C} \mathrm{NMR}\left(100 \mathrm{MHz}, \mathrm{CDCl}_{3}\right): \delta 146.5$, 139.0, 138.4, 128.9, 128.4, 126.8, 114.6, 97.6, 77.1, 37.3, 33.5, 25.0; HRMS (FAB) $[\mathrm{M}-\mathrm{H}]^{+}$calcd for $\mathrm{C}_{12} \mathrm{H}_{14} \mathrm{OI}: 301.0089$, found: 301.0109 . $; \mathrm{R}_{\mathrm{f}}=0.38$ (hexane/AcOEt $=4 / 1$ ).

\section{1-Iodo-2-(1-methoxymethoxy-hex-5-enyl)-benzene (1)}

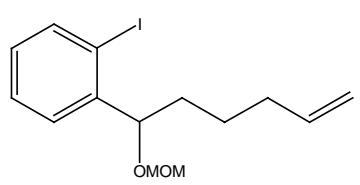

IR (thin film) $v_{\max }: 3064,2944,1642,1564,1464,1436,1152,1098,1032,1010,918,756 \mathrm{~cm}^{-1} ;{ }^{1} \mathrm{H}$ NMR (400 MHz, $\left.\mathrm{CDCl}_{3}\right): \delta 7.79(\mathrm{~d}, J=7.8 \mathrm{~Hz}, 1 \mathrm{H}), 7.41(\mathrm{~d} J=7.8 \mathrm{~Hz}, 1 \mathrm{H}), 7.35(\mathrm{dd}, J=7.8,7.2 \mathrm{~Hz}, 1 \mathrm{H}), 6.96$ $(\mathrm{dd}, J=7.8,7.2 \mathrm{~Hz}, 1 \mathrm{H}), 5.87-5.77(\mathrm{~m}, 1 \mathrm{H}), 5.02(\mathrm{~d}, J=17.1 \mathrm{~Hz}, 1 \mathrm{H}), 4.95(\mathrm{~d}, J=10.0 \mathrm{~Hz}, 1 \mathrm{H}), 4.84(\mathrm{dd}$, $J=4.8,4.8 \mathrm{~Hz}, 1 \mathrm{H}), 4.55(\mathrm{~d}, J=6.7 \mathrm{~Hz}, 1 \mathrm{H}), 4.48(\mathrm{~d}, J=6.7 \mathrm{~Hz}, 1 \mathrm{H}), 3.39(\mathrm{~s}, 3 \mathrm{H}), 2.17-2.08(\mathrm{~m}, 2 \mathrm{H})$, $1.72-1.65(\mathrm{~m}, 3 \mathrm{H}), 1.51(\mathrm{~m}, 1 \mathrm{H}) ;{ }^{13} \mathrm{C}$ NMR $\left(100 \mathrm{MHz}, \mathrm{CDCl}_{3}\right): \delta$ 144.6, 139.2, 138.5, 129.0, 128.4, 127.4, 114.6, 98.5, 94.7, 81.0, 55.9, 36.7, 33.5, 25.2; HRMS (FAB) $[\mathrm{M}]^{+}$calcd for $\mathrm{C}_{14} \mathrm{H}_{19} \mathrm{O}_{2} \mathrm{I}: 346.0430$, found: $346.0403 ; \mathrm{R}_{\mathrm{f}}=0.62($ hexane/AcOEt $=4 / 1)$.

\section{5-Methoxymethoxy-5,6,7,8,9,10-hexahydro-benzocyclooctene (2)}

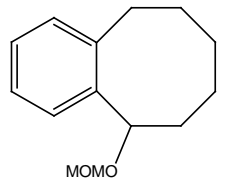

IR (thin film) $v_{\max }: 2932,1734,1452,1210,1154,1098,1040,758 \mathrm{~cm}^{-1} ;{ }^{1} \mathrm{H}$ NMR $\left(400 \mathrm{MHz}, \mathrm{CDCl}_{3}\right): \delta$ $7.44(\mathrm{~d}, J=7.6 \mathrm{~Hz}, 1 \mathrm{H}), 7.24$ (ddd, $J=7.6,7.3,1.7 \mathrm{~Hz}, 1 \mathrm{H}), 7.19$ (ddd, $J=7.3,7.3,1.7 \mathrm{~Hz}, 1 \mathrm{H}), 7.09$ (d, $J$ $=7.3 \mathrm{~Hz}, 1 \mathrm{H}), 5.06(\mathrm{dd}, J=4.6,4.6 \mathrm{~Hz}), 4.61(\mathrm{~d}, J=6.6 \mathrm{~Hz}, 1 \mathrm{H}), 4.58(\mathrm{~d}, J=6.6 \mathrm{~Hz}, 1 \mathrm{H}), 3.40(\mathrm{~s}, 3 \mathrm{H})$, 2.83-2.72 (m, 2H), 2.13-2.06 (m, 1H), 1.99-1.92 (m, 1H), 1.63-1.52 (m, 3H), 1.41-1.36 (m, 2H), 0.94-0.89 (m, 1H); ${ }^{13} \mathrm{C}$ NMR (100 MHz, $\left.\mathrm{CDCl}_{3}\right): \delta$ 140.7, 140.2, 128.9, 127.0, 126.3, 124.5, 94.3, 73.3, 55.5, 39.1, 32.7, 32.6, 26.6, 23.7; HRMS (FAB) $[\mathrm{M}+\mathrm{Na}]^{+}$calcd for $\mathrm{C}_{14} \mathrm{H}_{20} \mathrm{O}_{2} \mathrm{Na}$ : 243.1361, found: 243.1354. ; $\mathrm{R}_{\mathrm{f}}=$ 0.62 (hexane/AcOEt $=4 / 1)$.

\section{$d l$-carbonate (4)}

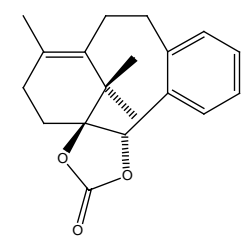


IR (KBr) $v_{\max }: 2940,1798,1286,1208,1020 \mathrm{~cm}^{-1} ;{ }^{1} \mathrm{H}$ NMR (300 MHz, $\left.\mathrm{CDCl}_{3}\right): \delta 7.45(\mathrm{~d}, J=7.5 \mathrm{~Hz}, 1 \mathrm{H})$, 7.29-7.18 (m, 3H), 5.57(s,1H), 2.96 (dd, $J=21.1,14.7 \mathrm{~Hz}, 1 \mathrm{H}), 2.68$ (dd, $J=21.1,14.7 \mathrm{~Hz}, 2 \mathrm{H}), 2.48-2.37$ (m, 1H), 2.34-2.24 (m, 2H), 2.20-1.90 (m, 1H), $1.45(\mathrm{~s}, 3 \mathrm{H}), 1.33-1.26(\mathrm{~m}, 1 \mathrm{H}), 1.18(\mathrm{~s}, 3 \mathrm{H}), 0.71(\mathrm{~s}, 3 \mathrm{H})$; ${ }^{13} \mathrm{C}$ NMR $\left(100 \mathrm{MHz}, \mathrm{CDCl}_{3}\right): \delta 154.2,138.6,135.0,134.4,131.1,130.3,128.0,126.2,123.7,92.5,79.5$, 39.8, 34.2, 28.8, 25.2, 23.0, 20.9, 19.7; HRMS (FAB) $[\mathrm{M}+\mathrm{H}]^{+}$calcd for $\mathrm{C}_{19} \mathrm{H}_{23} \mathrm{O}_{3}, 299.1647$, found,299.1650.; $\mathrm{R}_{\mathrm{f}}=0.60$ (hexane/AcOEt $=2 / 1$ ).

\section{$d l-(1 S, 2 S)-12,15,15-T r i m e t h y l-t r i c y c l o[9.3 .1 .03,8]$ pentadeca-3,5,7,11-tetraene-1,2-diol (5)}

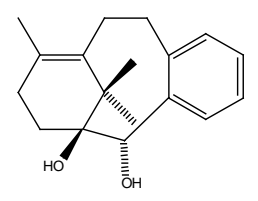

IR (KBr) $v_{\max }: 3390,2922,1458,1376,1020,750 \mathrm{~cm}^{-1} ;{ }^{1} \mathrm{H}$ NMR $\left(300 \mathrm{MHz}, \mathrm{CDCl}_{3}\right): \delta 7.62(\mathrm{~d}, J=7.3 \mathrm{~Hz}$, 1H), 7.20 (ddd, $J=7.3,7.3,1.7 \mathrm{~Hz}, 1 \mathrm{H}), 7.10$ (dd, $J=7.3,1.7 \mathrm{~Hz}, 1 \mathrm{H}), 7.03$ (ddd, $J=7.3,7.3,1.3 \mathrm{~Hz}$ ), $4.72(\mathrm{~s}, 1 \mathrm{H}), 2.86(\mathrm{~d}, J=1.5 \mathrm{~Hz}, 1 \mathrm{H}), 2.83(\mathrm{dd}, J=4.6,4.0 \mathrm{~Hz}, 1 \mathrm{H}), 2.79-2.68(\mathrm{~m}, 1 \mathrm{H}), 2.49$ (s, 1H), 2.43 (d, $J=4.4 \mathrm{~Hz}, 1 \mathrm{H}), 2.40(\mathrm{~d}, J=5.3 \mathrm{~Hz}, 1 \mathrm{H}), 2.37-2.31(\mathrm{~m}, 1 \mathrm{H}), 1.37(\mathrm{~s}, 3 \mathrm{H}), 1.33-1.19(\mathrm{~m}, 3 \mathrm{H}), 1.08$ (s, 3H), 0.47 (s, 3H); ${ }^{13} \mathrm{C} \mathrm{NMR}\left(100 \mathrm{MHz}, \mathrm{CDCl}_{3}\right): \delta 143.5,139.0,133.0,131.7,129.9,126.3,125.7,124.3$, 80.0, 71.4, 41.3, 34.4, 29.4, 29.3, 26.2, 25.7, 19.8, 19.3; HRMS (FAB) $[\mathrm{M}+\mathrm{Na}]^{+}$calcd for $\mathrm{C}_{18} \mathrm{H}_{24} \mathrm{O}_{2} \mathrm{Na}$, 295.1674, found, $295.1695 ; \mathrm{R}_{\mathrm{f}}=0.46$ (hexane/AcOEt $=2 / 1$ ).

\section{$d l$-(S)-((S)-3-iodo-1-(methoxymethoxy)-2,2,4-trimethylcyclohex-3-enyl)(2-vinylphenyl)methanol (b)}

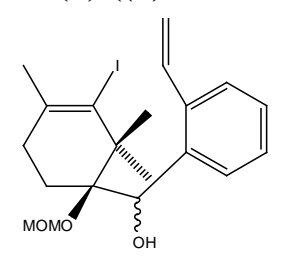

IR (thin film) $v_{\max }: 2924,1458,1388,1150,1022,890,826,770 \mathrm{~cm}^{-1} ;{ }^{1} \mathrm{H}$ NMR $\left(400 \mathrm{MHz}, \mathrm{CDCl}_{3}\right): \delta 7.68$ (d, $J=7.7 \mathrm{~Hz}, 0.09 \mathrm{H}), 7.57$ (d, $J=7.3 \mathrm{~Hz}, 0.91 \mathrm{H}), 7.44(\mathrm{~d}, J=7.7 \mathrm{~Hz}, 0.09 \mathrm{H}), 7.41(\mathrm{~d}, J=7.6 \mathrm{~Hz}, 0.91 \mathrm{H})$, 7.32-7.23 (m, 2H), $5.60(\mathrm{~d}, J=17.3 \mathrm{~Hz}, 0.91 \mathrm{H}), 5.58(\mathrm{~d}, J=17.3 \mathrm{~Hz}, 0.09 \mathrm{H}), 5.38(\mathrm{~d}, J=3.9 \mathrm{~Hz}, 0.09 \mathrm{H})$, $5.32(\mathrm{~d}, J=11.0 \mathrm{~Hz}, 0.91 \mathrm{H}), 5.30(\mathrm{~d}, J=11.0 \mathrm{~Hz}, 0.09 \mathrm{H}), 5.20(\mathrm{~d}, J=8.5 \mathrm{~Hz}, 0.91 \mathrm{H}), 4.93(\mathrm{~d}, J=6.8 \mathrm{~Hz}$, $0.91 \mathrm{H}), 4.79(\mathrm{~d}, J=6.8 \mathrm{~Hz}, 0.91 \mathrm{H}), 4.17(\mathrm{~d}, J=7.5 \mathrm{~Hz}, 0.09 \mathrm{H}), 4.13(\mathrm{~d}, J=7.5 \mathrm{~Hz}, 0.09 \mathrm{H}), 3.53(\mathrm{~s}, 2.73 \mathrm{H})$, 3.25 (s, 0.27H), 2.03-1.85 (m, 1H), $1.93(\mathrm{~s}, 0.27 \mathrm{H}), 1.90-1.86(\mathrm{~m}, 1 \mathrm{H}), 1.83(\mathrm{~s}, 2.73 \mathrm{H}), 1.76-1.69(\mathrm{~m}, 1 \mathrm{H})$, 1.65-1.60 (m, 1H), $1.34(\mathrm{~s}, 2.73 \mathrm{H}), 1.30(\mathrm{~s}, 2.73 \mathrm{H}), 1.24(\mathrm{~s}, 0.27 \mathrm{H}), 1.22(\mathrm{~s}, 0.27 \mathrm{H})$; HRMS (FAB) $[\mathrm{M}+\mathrm{Na}]^{+}$cacld for $\mathrm{C}_{20} \mathrm{H}_{27} \mathrm{O}_{3} \mathrm{I}, 465.0903$, found, $465.0922 ; \mathrm{R}_{\mathrm{f}}=0.42$ (hexane/AcOEt $=4 / 1$ ). 


\section{1-Hydroxymethyl-3-iodo-2,2,4-trimethyl-cyclohex-3-enol (7)}

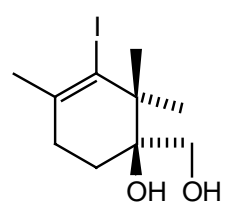

IR (thin film) $v_{\max }: 3410,2975,1457,1375,1202,1102,1043,894,661 \mathrm{~cm}^{-1} ;{ }^{1} \mathrm{H}$ NMR $\left(400 \mathrm{MHz}, \mathrm{CDCl}_{3}\right)$ : $\delta 3.76(\mathrm{~d}, J=11.0 \mathrm{~Hz}, 1 \mathrm{H}), 3.53-3.50(\mathrm{~m}, 1 \mathrm{H}), 2.33(\mathrm{dt}, J=17.8,6.6 \mathrm{~Hz}, 1 \mathrm{H}), 2.21(\mathrm{dd}, J=6.4,6.1 \mathrm{~Hz}$, 1H), 2.16 (br, 1H), 2.09 (br, 1H), $1.90(\mathrm{~s}, 3 \mathrm{H}), 1.88-1.76(\mathrm{~m}, 2 \mathrm{H}), 1.20(\mathrm{~s}, 3 \mathrm{H}), 1.06(\mathrm{~s}, 3 \mathrm{H}) ;{ }^{13} \mathrm{C}$ NMR (100 $\mathrm{MHz}, \mathrm{CDCl}_{3}$ ): $\delta 137.3,115.8,74.6,65.6,45.7,31.0,30.5,26.7,26.5,26.1 ; \mathrm{HRMS}(\mathrm{FAB})[\mathrm{M}+\mathrm{Na}]^{+}$calcd for $\mathrm{C}_{10} \mathrm{H}_{17} \mathrm{IO}_{2} \mathrm{Na}, 319.0171$, found, 319.0183; $\mathrm{R}_{\mathrm{f}}=0.29$ (hexane/AcOEt = 1 / 1).

\section{Acetic acid 1-hydroxy-3-iodo-2,2,4-trimethyl-cyclohex-3-enylmethyl ester (c)}

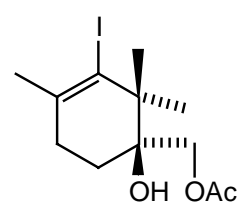

IR (thin film) $v_{\max }: 3492,2976,1742,1458,1384,1246,1038,896 \mathrm{~cm}^{-1} ;{ }^{1} \mathrm{H}$ NMR $\left(400 \mathrm{MHz}, \mathrm{CDCl}_{3}\right): \delta$ $4.28(\mathrm{~d}, J=11.7 \mathrm{~Hz}, 1 \mathrm{H}), 4.10(\mathrm{~d}, J=11.7 \mathrm{~Hz}, 1 \mathrm{H}), 2.31(\mathrm{dt}, J=17.8,6.1 \mathrm{~Hz}, 1 \mathrm{H}), 2.19-2.15(\mathrm{~m}, 1 \mathrm{H}), 2.21$ $(\mathrm{s}, 3 \mathrm{H}), 1.90(\mathrm{~s}, 3 \mathrm{H}), 1.87-1.74(\mathrm{~m}, 2 \mathrm{H}), 1.21(\mathrm{~s}, 3 \mathrm{H}), 1.13(\mathrm{~s}, 3 \mathrm{H}) ;{ }^{13} \mathrm{C}$ NMR $\left(100 \mathrm{MHz}, \mathrm{CDCl}_{3}\right): \delta 171.3$, 137.2, 114.9, 73.5, 68.0, 45.8, 30.9, 30.6, 27.2, 26.9, 25.8, 21.0; HRMS (FAB) $[\mathrm{M}+\mathrm{H}]^{+}$calcd for $\mathrm{C}_{12} \mathrm{H}_{19} \mathrm{IO}_{3}$, 339.0457, found, 339.0444; $\mathrm{R}_{\mathrm{f}}=0.51$ (hexane/ $\mathrm{AcOEt}=1 / 1$ ).

\section{Acetic acid 3-iodo-1-methoxymethoxy-2,2,4-trimethyl-cyclohex-3-enylmethyl ester (d)}

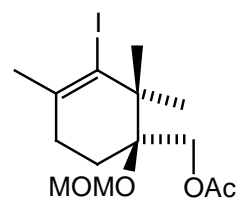

IR (thin film) $v_{\max }: 2984,1748,1458,1382,1234,1152,1108,1044,920,894 \mathrm{~cm}^{-1} ;{ }^{1} \mathrm{H}$ NMR $(400 \mathrm{MHz}$, $\left.\mathrm{CDCl}_{3}\right): \delta 4.83(\mathrm{~d}, J=7.3 \mathrm{~Hz}, 1 \mathrm{H}), 4.79(\mathrm{~d}, J=7.3 \mathrm{~Hz}, 1 \mathrm{H}), 4.28(\mathrm{~d}, J=12.2 \mathrm{~Hz}, 1 \mathrm{H}), 4.21(\mathrm{~d}, J=12.2 \mathrm{~Hz}$, 1H), 2.27-2.10 (m, 3H), 2.08 (s, 3H), 1.95-1.89 (m, 1H), 1.87 (s, 3H), $1.19(\mathrm{~s}, 3 \mathrm{H}), 1.15(\mathrm{~s}, 3 \mathrm{H}) ;{ }^{13} \mathrm{C}$ NMR $\left(100 \mathrm{MHz}, \mathrm{CDCl}_{3}\right): \delta$ 170.7, 136.9, 114.6, 91.5, 78.2, 67.4, 55.6, 46.8, 31.0, 30.6, 27.2, 25.7, 23.1, 21.1; HRMS (FAB) $[\mathrm{M}+\mathrm{H}]^{+}$calcd for $\mathrm{C}_{14} \mathrm{H}_{24} \mathrm{IO}_{4}, 383.0719$, found, 383.0742; $\mathrm{R}_{\mathrm{f}}=0.67$ (hexane/ $\mathrm{AcOEt}=1 / 1$ ). 
(3-Iodo-1-methoxymethoxy-2,2,4-trimethyl-cyclohex-3-enyl)-methanol (e)

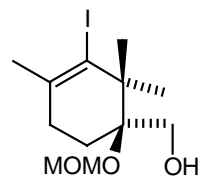

IR (thin film) $v_{\max }: 3476,2980,1464,1360,1148,1106,1042,894,668 \mathrm{~cm}^{-1} ;{ }^{1} \mathrm{H}$ NMR (400 MHz, $\left.\mathrm{CDCl}_{3}\right)$ : $\delta 4.96(\mathrm{~d}, J=7.7 \mathrm{~Hz}, 1 \mathrm{H}), 4.57(\mathrm{~d}, J=7.7 \mathrm{~Hz}, 1 \mathrm{H}), 3.71(\mathrm{~d}, J=10.1 \mathrm{~Hz}, 1 \mathrm{H}), 3.58(\mathrm{~d}, J=10.1 \mathrm{~Hz}, 1 \mathrm{H})$, 2.29-2.20 (m, 2H), 2.13-2.07 (m, 1H), $1.89(\mathrm{~s}, 3 \mathrm{H}), 1.75-1.68(\mathrm{~m}, 1 \mathrm{H}), 1.16(\mathrm{~s}, 3 \mathrm{H}), 1.11(\mathrm{~s}, 3 \mathrm{H}) ;{ }^{13} \mathrm{C} \mathrm{NMR}$ $\left(100 \mathrm{MHz}_{\mathrm{CDCl}}\right): \delta 136.9,115.2,90.4,81.3,64.6,55.6,46.3,30.8,30.6,27.0,26.0,21.2 ;$ HRMS (FAB) $[\mathrm{M}+\mathrm{H}]^{+}$calcd for $\mathrm{C}_{12} \mathrm{H}_{22} \mathrm{IO}_{3}, 341.0613$, found, 341.0602; $\mathrm{R}_{\mathrm{f}}=0.53$ (hexane/ AcOEt = $2 / 1$ ).

\section{(S)-3-Iodo-1-methoxymethoxy-2,2,4-trimethyl-cyclohex-3-enecarbaldehyde $((+)-8)$}

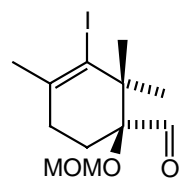

$[\alpha]^{27.4}+76.2$ (c $\left.0.98, \mathrm{CHCl}_{3}\right)$; IR (thin film) $v_{\max }: 2889,1724,1458,1154,1023,917,776 \mathrm{~cm}^{-1} ;{ }^{1} \mathrm{H}$ NMR (400 MHz, $\left.\mathrm{CDCl}_{3}\right): \delta 9.76(\mathrm{~s}, 1 \mathrm{H}), 4.75(\mathrm{~d}, J=7.3 \mathrm{~Hz}, 1 \mathrm{H}), 4.64(\mathrm{~d}, J=7.3 \mathrm{~Hz}, 1 \mathrm{H}), 2.25-2.21(\mathrm{~m}$, 2H), 2.12-2.04 (m, 1H), $1.94(\mathrm{dd}, J=5.4,4.4 \mathrm{~Hz}, 1 \mathrm{H}), 1.91(\mathrm{~s}, 3 \mathrm{H}), 1.38(\mathrm{~s}, 3 \mathrm{H}), 1.06(\mathrm{~s}, 3 \mathrm{H}) ;{ }^{13} \mathrm{C} \mathrm{NMR}$ $\left(100 \mathrm{MHz} \mathrm{CDCl}_{3}\right): \delta 202.9,137.1,114.1,91.9,83.4,56.0,45.3,30.9,29.2,27.3,26.6,20.4 ;$ HRMS (FAB) $[\mathrm{M}+\mathrm{H}]^{+}$calcd for $\mathrm{C}_{12} \mathrm{H}_{20} \mathrm{IO}_{3}, 339.0457$, found, 339.0451; $\mathrm{R}_{\mathrm{f}}=0.60$ (hexane/ AcOEt = $2 / 1$ ).

dl-1-((S)-(benzyloxy)((S)-3-iodo-1-(methoxymethoxy)-2,2,4-trimethylcyclohex-3-enyl)methyl)-2-vinyl benzene (9)

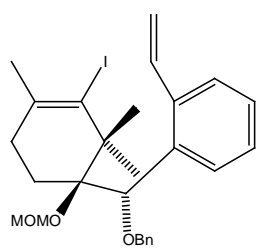

IR (thin film) $v_{\max }: 2932,1738,1454,1374,1242,1150,1102,1028,918,700 \mathrm{~cm}^{-1} ;{ }^{1} \mathrm{H}$ NMR $(400 \mathrm{MHz}$, $\left.\mathrm{CDCl}_{3}\right): \delta$ 7.75-7.68 (br, 1H), 7.46-7.38 (br, 1H), 7.28-7.15 (m, 7H), 7.01-6.94 (m, 1H), $5.68(\mathrm{~d}, J=17.1$ $\mathrm{Hz}, 1 \mathrm{H}), 5.35$ (d, J = 10.7 Hz, 1H), 4.90 (s, 1H), 4.75 (d, J=7.1 Hz, 1H), 4.56 (d, J= 7.1 Hz, 1H), 4.27 (d, $J=11.2 \mathrm{~Hz}, 1 \mathrm{H}), 4.15(\mathrm{~d}, J=11.2 \mathrm{~Hz}, 1 \mathrm{H}), 3.29(\mathrm{~s}, 3 \mathrm{H}), 2.44-2.40(\mathrm{~m}, 1 \mathrm{H}), 1.97-1.93(\mathrm{~m}, 1 \mathrm{H}), 1.85$ (s, $1 \mathrm{H}), 1.72-1.69(\mathrm{~m}, 1 \mathrm{H}), 1.28(\mathrm{~s}, 1 \mathrm{H}), 1.15(\mathrm{~s}, 3 \mathrm{H}) ;{ }^{13} \mathrm{C} \mathrm{NMR}\left(100 \mathrm{MHz}, \mathrm{CDCl}_{3}\right): \delta 138.4,137.8,136.6$, 136.5, 134.7, 129.4, 128.2, 128.1, 127.9, 127.7, 127.5, 125.7, 118.6, 116.9, 92.4, 82.9, 79.8, 70.1, 55.7, 48.2, 31.3, 29.7, 29.4, 27.4, 24.2; HRMS (FAB) $[\mathrm{M}+\mathrm{Na}]^{+}$calcd for $\mathrm{C}_{27} \mathrm{H}_{33} \mathrm{O}_{3} \mathrm{INa}$,555.1372, found, $555.1390 ; \mathrm{R}_{\mathrm{f}}$ $=0.60($ hexane/AcOEt $=4 / 1)$. 
d1-1-((R)-(benzyloxy)((S)-3-iodo-1-(methoxymethoxy)-2,2,4-trimethylcyclohex-3-enyl)methyl)-2-vinyl benzene (f)

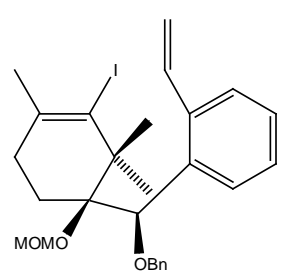

IR (thin film) $v_{\max }: 2928,1454,1360,1150,1070,1044,1022,920,886 \mathrm{~cm}^{-1} ;{ }^{1} \mathrm{H}$ NMR $\left(400 \mathrm{MHz}, \mathrm{CDCl}_{3}\right)$ : $\delta$ 7.57-7.50 (m, 2H), 7.37-7.20 (m, 8H), $5.63(\mathrm{~d}, J=17.3 \mathrm{~Hz}, 1 \mathrm{H}), 5.34(\mathrm{~d}, J=10.7 \mathrm{~Hz}, 1 \mathrm{H}), 5.15(\mathrm{~s}, 1 \mathrm{H})$, $4.11(\mathrm{~s}, 2 \mathrm{H}), 3.99$ (d, $J=7.8 \mathrm{~Hz}, 1 \mathrm{H}), 3.87(\mathrm{~d}, J=7.8 \mathrm{~Hz}, 1 \mathrm{H}), 3.14(\mathrm{~s}, 3 \mathrm{H}), 2.54-2.46(\mathrm{~m}, 1 \mathrm{H}), 2.37-2.31$ (m, 1H), 2.27-2.19 (m, 1H), 2.13-2.06 (m, 1H), $1.82(\mathrm{~s}, 3 \mathrm{H}), 1.33$ (s, 3H), 1.29 (s, 3H); ${ }^{13} \mathrm{C}$ NMR (100 $\left.\mathrm{MHz}, \mathrm{CDCl}_{3}\right): \delta 139.3,138.1,136.6,136.2,135.4,128.3,128.1,127.8,127.5,127.3,127.2,126.2,116.3$, $115.7,91.1,82.6,79.8,70.5,55.8,47.8,31.3,29.7,28.6,28.0,20.4$; HRMS (FAB) $[\mathrm{M}+\mathrm{H}]^{+}$calcd for $\mathrm{C}_{27} \mathrm{H}_{34} \mathrm{O}_{3} \mathrm{I}, 533.1553$, found, $533.1543 ; \mathrm{R}_{\mathrm{f}}=0.67$ (hexane/AcOEt $=4 / 1$ ).

$d l$-acetonide (11)

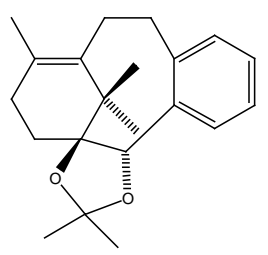

IR (KBr) $v_{\max }: 2983,1458,1373,1240,1223,1087,1048 \mathrm{~cm}^{-1} ;{ }^{1} \mathrm{H}$ NMR $\left(300 \mathrm{MHz}, \mathrm{CDCl}_{3}\right): \delta 7.56$ (d, $J=$ $7.7 \mathrm{~Hz}, 1 \mathrm{H}), 7.19$ (dd, $J=7.7,7.3 \mathrm{~Hz}, 1 \mathrm{H}), 7.14(\mathrm{~d}, J=7.0 \mathrm{~Hz}, 1 \mathrm{H}), 7.07$ (dd, $J=7.3,7.0 \mathrm{~Hz}, 1 \mathrm{H}), 4.94$ (s, 1H), $2.86(\mathrm{dd}, J=13.4,7.7 \mathrm{~Hz}, 1 \mathrm{H}), 2.77(\mathrm{ddd}, J=13.4,11.8,7.0 \mathrm{~Hz}, 1 \mathrm{H}), 2.60(\mathrm{ddd}, J=12.7,11.8,7.7$ $\mathrm{Hz}, 1 \mathrm{H}), 2.41$ (dd, $J=12.7,7.0 \mathrm{~Hz}, 1 \mathrm{H}), 2.21$ (ddd, 13.2, 12.2, $4.4 \mathrm{~Hz}, 2 \mathrm{H}), 1.68-1.62(\mathrm{~m}, 1 \mathrm{H}), 1.57$ (s, 3H), $1.41(\mathrm{~s}, 3 \mathrm{H}), 1.40(\mathrm{~s}, 3 \mathrm{H}), 1.21-1.13(\mathrm{~m}, 1 \mathrm{H}), 1.10(\mathrm{~s}, 3 \mathrm{H}), 0.64(\mathrm{~s}, 3 \mathrm{H}) ;{ }^{13} \mathrm{C} \mathrm{NMR}\left(100 \mathrm{MHz}, \mathrm{CDCl}_{3}\right): \delta$ 139.0, 138.4, 132.9, 131.8, 130.5, 126.6, 125.7, 124.2, 105.8, 87.6, 76.8, 39.8, 34.4, 29.4, 29.0, 28.5, 26.6, 25.8, 25.7, 20.6, 19.7; HRMS (FAB) $[M]^{+}$calcd for $\mathrm{C}_{21} \mathrm{H}_{28} \mathrm{O}_{2}, 312.2089$, found, $312.2068 ; \mathrm{R}_{\mathrm{f}}=0.43$ (hexane/AcOEt $=10 / 1)$.

$d l-(1 S, 2 S)$-2-Benzyloxy-1-methoxymethoxy-12,15,15-trimethyl-tricyclo[9.3.1.03,8]pentadeca-3,5,7,11-t etraene (12) 


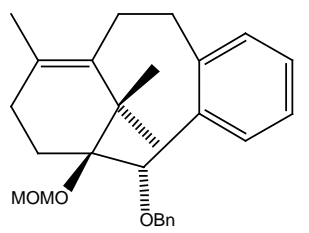

IR (thin film) $v_{\max }: 2928,1458,1384,1154,1110,1066,1030,918,758,738,698 \mathrm{~cm}^{-1} ;{ }^{1} \mathrm{H}$ NMR $(400$ $\left.\mathrm{MHz}, \mathrm{CDCl}_{3}\right): \delta 7.57(\mathrm{~d}, J=7.4 \mathrm{~Hz}, 1 \mathrm{H}), 7.31-7.25(\mathrm{~m}, 5 \mathrm{H}), 7.14(\mathrm{dd}, J=8.1,7.0 \mathrm{~Hz}, 1 \mathrm{H}), 7.11(\mathrm{~d}, J=7.4$ Hz, 1H), $7.02(\mathrm{dd}, J=8.1,7.0 \mathrm{~Hz}, 1 \mathrm{H}), 5.28$ (d, $J=7.9 \mathrm{~Hz}, 1 \mathrm{H}), 4.66$ (d, $J=7.9 \mathrm{~Hz}, 1 \mathrm{H}), 4.39$ (s, 1H), 4.17 (d, $J=2.9 \mathrm{~Hz}, 2 \mathrm{H}), 2.47-2.31(\mathrm{~m}, 3 \mathrm{H}), 2.27-2.19(\mathrm{~m}, 1 \mathrm{H}), 2.11-2.04(\mathrm{~m}, 1 \mathrm{H}), 1.38(\mathrm{~s}, 3 \mathrm{H}), 1.36-1.33(\mathrm{~m}$, $1 \mathrm{H}), 1.08(\mathrm{~s}, 3 \mathrm{H}), 0.43(\mathrm{~s}, 3 \mathrm{H}) ;{ }^{13} \mathrm{C} \mathrm{NMR}\left(100 \mathrm{MHz}, \mathrm{CDCl}_{3}\right): \delta 142.2,140.2,138.6,132.9,131.7,129.9$, 128.1, 127.5, 127.1, 126.2, 125.7, 125.0, 93.8, 85.0, 81.3, 71.1, 55.4, 42.3, 34.4, 29.5, 29.2, 26.8, 20.4, 19.6, 19.5; HRMS (FAB) $[\mathrm{M}+\mathrm{Na}]^{+}$calcd for $\mathrm{C}_{27} \mathrm{H}_{34} \mathrm{O}_{3} \mathrm{Na}$, 424.2406, found, $429.2435 ; \mathrm{R}_{\mathrm{f}}=0.66$ (hexane/AcOEt $=4 / 1)$.

\section{$(2 R, 4 a R, 8 \mathrm{a} S)$-hexahydro-4a-methyl-2-phenylbenzo[d][1,3]dioxin-5-one (14)}

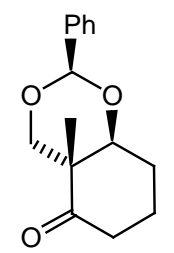

$[\alpha]^{28.2}+73.0\left(\mathrm{c} 1.03, \mathrm{CHCl}_{3}\right) ; \mathrm{mp} 71-73{ }^{\circ} \mathrm{C}$; IR (KBr) $v_{\max }: 2953,1703,1378,1216,1067,764,696 \mathrm{~cm}^{-1}$; ${ }^{1} \mathrm{HNMR}\left(400 \mathrm{MHz}, \mathrm{CDCl}_{3}\right): \delta$ 7.50-7.49 (m, 2H), 7.39-7.33 (m, 3H), $5.49(\mathrm{~s}, 1 \mathrm{H}), 4.11(\mathrm{~d}, J=11.5 \mathrm{~Hz}, 1 \mathrm{H})$, $4.02(\mathrm{~d}, J=11.5 \mathrm{~Hz}, 1 \mathrm{H}), 3.86$ (dd, $J=11.2,4.4 \mathrm{~Hz}, 1 \mathrm{H}), 2.65$ (ddd, $J=14.9,14.4,6.6 \mathrm{~Hz}, 1 \mathrm{H}), 2.22$ (dd, $J=14.9,4.7 \mathrm{~Hz}, 1 \mathrm{H}), 2.08-1.92(\mathrm{~m}, 3 \mathrm{H}), 1.72-1.60(\mathrm{~m}, 1 \mathrm{H}), 1.50(\mathrm{~s}, 3 \mathrm{H}) ;{ }^{13} \mathrm{C} \mathrm{NMR}\left(100 \mathrm{MHz}, \mathrm{CDCl}_{3}\right): \delta$ $212.1,137.8,129.0,128.3,126.1,102.4,82.2,73.3,48.7,36.9,25.5,21.0,16.1$; HRMS (FAB) $[\mathrm{M}+\mathrm{H}]^{+}$ calcd for $\mathrm{C}_{15} \mathrm{H}_{19} \mathrm{O}_{3}, 247.1334$, found, 247.1318; $\mathrm{R}_{\mathrm{f}}=0.69$ (hexane / AcOEt = $3 / 1$ ).

$(2 R, 4 \mathrm{a} R, 8 \mathrm{a} S)-4 \mathrm{a}, 7,8,8 \mathrm{a}-$-tetrahydro-5-iodo-4a-methyl-2-phenyl-4H-benzo[d][1,3]dioxine (15)<smiles>P[C@@H]1CO[C@@H]2C(I)=CCC[C@@H]2O1</smiles>

$[\alpha]^{28.8}{ }_{\mathrm{D}}-66.6$ (c 1.03, $\mathrm{CHCl}_{3}$ ); $\mathrm{mp} 54-56^{\circ} \mathrm{C}$; IR (KBr) $v_{\max }: 1459,1375,1225,1092,909,754,696 \mathrm{~cm}^{-1}$; ${ }^{1} \mathrm{HNMR}\left(400 \mathrm{MHz}, \mathrm{CDCl}_{3}\right): \delta 7.52-7.50(\mathrm{~m}, 2 \mathrm{H}), 7.40-7.32(\mathrm{~m}, 3 \mathrm{H}), 6.28(\mathrm{dd}, J=3.4,3.2 \mathrm{~Hz}, 1 \mathrm{H}), 5.54$ (s, 1H), 3.93 (t, $J=8.1 \mathrm{~Hz}, 1 \mathrm{H}), 3.89$ (d, $J=10.6 \mathrm{~Hz}, 1 \mathrm{H}), 3.58(\mathrm{~d}, J=10.6 \mathrm{~Hz}, 1 \mathrm{H}), 2.35-2.15(\mathrm{~m}, 2 \mathrm{H})$, 1.94-1.88 (m, 2H), 1.32 (s, 3H); ${ }^{13} \mathrm{C}$ NMR (100 MHz, $\left.\mathrm{CDCl}_{3}\right): \delta$ 137.8, 137.7, 129.0, 128.3, 126.2, 103.2, 
103.1, 81.1, 80.1, 40.8, 28.4, 23.1, 18.0; HRMS (FAB) $[\mathrm{M}+\mathrm{H}]^{+}$calcd for $\mathrm{C}_{15} \mathrm{H}_{18} \mathrm{O}_{2} \mathrm{I}, 357.0352$, found, $357.0338 ; \mathrm{R}_{\mathrm{f}}=0.31$ (hexane $/ \mathrm{AcOEt}=3 / 1$ ).

((1R,6S)-6-(benzyloxy)-2-iodo-1-methylcyclohex-2-enyl)methanol (g)

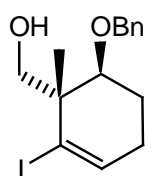

$[\alpha]^{27.4}$ D -28.4 (c 0.99, $\mathrm{CHCl}_{3}$ ); IR (thin film) $v_{\max }: 3456,2944,2880,1454,1102,1042,734,698 \mathrm{~cm}^{-1}$; ${ }^{1} \mathrm{HNMR}\left(400 \mathrm{MHz}, \mathrm{CDCl}_{3}\right): \delta 7.35-7.27(\mathrm{~m}, 5 \mathrm{H}), 6.47(\mathrm{t}, J=3.9 \mathrm{~Hz}, 1 \mathrm{H}), 4.68(\mathrm{~d}, J=11.7 \mathrm{~Hz}, 1 \mathrm{H}), 4.50(\mathrm{~d}$, $J=11.7 \mathrm{~Hz}, 1 \mathrm{H}), 3.91(\mathrm{dd}, J=12.1,3.3 \mathrm{~Hz}, 1 \mathrm{H}), 3.62(\mathrm{dd}, J=10.5,8.6 \mathrm{~Hz}, 1 \mathrm{H}), 3.49(\mathrm{~d}, J=10.5 \mathrm{~Hz}, 1 \mathrm{H})$, $2.12(\mathrm{dt}, J=8.3,3.9 \mathrm{~Hz}, 2 \mathrm{H}), 2.02(\mathrm{dt}, J=12.1,3.9 \mathrm{~Hz}, 1 \mathrm{H}), 1.80-1.70(\mathrm{~m}, 1 \mathrm{H}), 1.44(\mathrm{br}, 1 \mathrm{H}), 0.97(\mathrm{~s}, 3 \mathrm{H})$; ${ }^{13} \mathrm{C}$ NMR $\left(100 \mathrm{MHz}, \mathrm{CDCl}_{3}\right): \delta 140.9,138.5,128.3,127.6,109.3,75.1,71.5,68.8,49.3,28.5,22.5,17.6$; HRMS (FAB) $[\mathrm{M}+\mathrm{H}]^{+}$calcd for, $\mathrm{C}_{15} \mathrm{H}_{20} \mathrm{O}_{2} \mathrm{I}, 359.0508$, found, 359.0487; $\mathrm{Rf}=0.44$ (hexane/AcOEt = $3 / 1$ ).

(1S,6S)-6-(benzyloxy)-2-iodo-1-methylcyclohex-2-enecarbaldehyde (h)

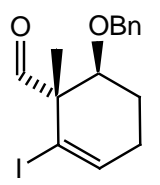

$[\alpha]^{24.3}+33.9\left(\mathrm{c} 1.05, \mathrm{CHCl}_{3}\right) ;$ IR (thin film) $v_{\max }: 2940,2332,1734,1456,1366,1100,1028,738,698$ $\mathrm{cm}^{-1} ;{ }^{1} \mathrm{H}$ NMR $\left(400 \mathrm{MHz}, \mathrm{CDCl}_{3}\right): \delta 9.31(\mathrm{~s}, 1 \mathrm{H}), 7.35-7.26(\mathrm{~m}, 5 \mathrm{H}), 6.60(\mathrm{dd}, J=4.7,3.3 \mathrm{~Hz}, 1 \mathrm{H}), 4.57$ $(\mathrm{d}, J=12.0 \mathrm{~Hz}, 1 \mathrm{H}), 4.42(\mathrm{~d}, J=12.0 \mathrm{~Hz}, 1 \mathrm{H}), 3.92(\mathrm{dd}, J=10.3,3.2 \mathrm{~Hz}, 1 \mathrm{H}), 2.30-2.21(\mathrm{~m}, 1 \mathrm{H})$, 2.18-2.09 (m, 1H), 2.01-1.94 (m, 1H), 1.84-1.74 (m,1H), $1.36(\mathrm{~s}, 3 \mathrm{H}) ;{ }^{13} \mathrm{C}$ NMR $\left(100 \mathrm{MHz}, \mathrm{CDCl}_{3}\right): \delta$ $201.2,140.9,137.8,128.3,127.7,127.6,97.4,75.9,71.3,58.8,27.8,22.0,16.6$; HRMS (FAB) $[\mathrm{M}+\mathrm{H}]^{+}$ calcd for $\mathrm{C}_{15} \mathrm{H}_{18} \mathrm{O}_{2} \mathrm{I}, 357.0352$, found, 357.0322; $\mathrm{R}_{\mathrm{f}}=0.56$ (hexane/ AcOEt $=3 / 1$ ).

\section{1-(((1S,2R)-3-iodo-2-methyl-2-vinylcyclohex-3-enyloxy)methyl)benzene (16)}

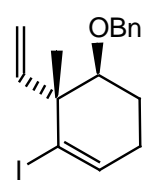

$[\alpha]^{27.3}+60.1$ (c 0.61, $\mathrm{CHCl}_{3}$ ); IR (thin film) $v_{\max }: 2944,2872,1454,1098,1074,924,736,698 \mathrm{~cm}^{-1} ;{ }^{1} \mathrm{H}$ NMR (400 MHz, $\left.\mathrm{CDCl}_{3}\right): \delta 7.33-7.26(\mathrm{~m}, 5 \mathrm{H}), 6.49(\mathrm{t}, J=4.0 \mathrm{~Hz}, 1 \mathrm{H}), 5.64(\mathrm{dd}, J=17.3,10.5 \mathrm{~Hz}, 1 \mathrm{H})$, $5.22(\mathrm{~d}, J=10.5 \mathrm{~Hz}, 1 \mathrm{H}), 5.15(\mathrm{~d}, J=17.3 \mathrm{~Hz}, 1 \mathrm{H}), 4.60(\mathrm{~d}, J=11.8 \mathrm{~Hz}, 1 \mathrm{H}), 4.47(\mathrm{~d}, J=11.8 \mathrm{~Hz}, 1 \mathrm{H})$, $3.53(\mathrm{dd}, J=7.4,2.6 \mathrm{~Hz}, 1 \mathrm{H}), 2.26-2.17(\mathrm{~m}, 1 \mathrm{H}), 2.04-1.95(\mathrm{~m}, 1 \mathrm{H}), 1.93(\mathrm{~m}, 2 \mathrm{H}), 1.28(\mathrm{~s}, 3 \mathrm{H}) ;{ }^{13} \mathrm{C} \mathrm{NMR}$ $\left(100 \mathrm{MHz}, \mathrm{CDCl}_{3}\right): \delta 144.2,138.8,138.5,128.2,127.6,127.4,115.7,109.0,79.6,71.8,50.2,26.8,22.9$, 
21.8; HRMS (FAB) $[\mathrm{M}+\mathrm{H}]^{+}$calcd for $\mathrm{C}_{16} \mathrm{H}_{20} \mathrm{OI}, 355.0559$, found, 355.0571; $\mathrm{R}_{\mathrm{f}}=0.63$ (benzene/ AcOEt $=$ $20 / 1)$.

(S)-((5S,6S)-5-benzyloxy-6-methyl-6-vinylcyclohex-1-enyl)((S)-3-iodo-1-(methoxymethoxy)-2,2,4-trim ethylcyclohex-3-enyl)methanol (i)

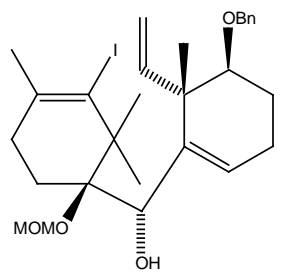

$[\alpha]_{D}^{20.4}+28.1$ ( c 0.32, $\mathrm{CHCl}_{3}$ ); IR (thin film) $v_{\max }: 3572,2948,1456,1158,1094,1028,918,886,788$ $\mathrm{cm}^{-1}$; ${ }^{1} \mathrm{H} \mathrm{NMR}\left(400 \mathrm{MHz}, \mathrm{CDCl}_{3}\right): \delta 7.32(\mathrm{~d}, J=4.2 \mathrm{~Hz}, 4 \mathrm{H}), 7.27-7.23(\mathrm{~m}, 1 \mathrm{H}), 6.10(\mathrm{dd}, J=4.9,2.9 \mathrm{~Hz}$, 1H), $5.64(\mathrm{dd}, J=17.4,10.6 \mathrm{~Hz}, 1 \mathrm{H}), 5.22(\mathrm{~d}, J=17.4 \mathrm{~Hz}, 1 \mathrm{H}), 5.19$ (d, $J=10.6 \mathrm{~Hz}, 1 \mathrm{H}), 4.92(\mathrm{~d}, J=6.8$ $\mathrm{Hz}, 1 \mathrm{H}), 4.71(\mathrm{~d}, J=6.8 \mathrm{~Hz}, 1 \mathrm{H}), 4.62(\mathrm{~d}, J=2.0 \mathrm{~Hz}, 1 \mathrm{H}), 4.48(\mathrm{~d}, J=2.0 \mathrm{~Hz}, 1 \mathrm{H}), 4.06(\mathrm{~d}, J=9.7 \mathrm{~Hz}$, 1H), 3.43-3.38 (m, 1H), $3.41(\mathrm{~s}, 3 \mathrm{H}), 2.98(\mathrm{~d}, J=9.7 \mathrm{~Hz}, 1 \mathrm{H}), 2.38-1.66(\mathrm{~m}, 8 \mathrm{H}), 1.85(\mathrm{~s}, 3 \mathrm{H}), 1.32(\mathrm{~s}, 3 \mathrm{H})$, $1.31(\mathrm{~s}, 3 \mathrm{H}), 1.17(\mathrm{~s}, 3 \mathrm{H}) ;{ }^{13} \mathrm{C} \mathrm{NMR}\left(100 \mathrm{MHz}, \mathrm{CDCl}_{3}\right) \delta 145.0,144.6,139.0,136.8,128.0,127.3,127.1$, 124.6, 117.8, 115.6, 92.7, 82.7, 81.7, 71.4, 71.3, 56.3, 48.7, 47.7, 31.2, 30.6, 28.9, 28.6, 25.4, 24.2, 22.4, 18.1; HRMS (FAB) $[\mathrm{M}+\mathrm{H}]^{+}$calcd for $\mathrm{C}_{28} \mathrm{H}_{40} \mathrm{IO}_{4}, 567.1971$, found, 567.1945; $\mathrm{Rf}=0.24$ (hexane/ AcOEt $=$ $4 / 1)$.

1-(((S)-((5S,6S)-5-benzyloxy-6-methyl-6-vinylcyclohex-1-enyl)((S)-3-iodo-1-(methoxymethoxy)-2,2,4-t rimethylcyclohex-3-enyl)methoxy)methyl)benzene (17)

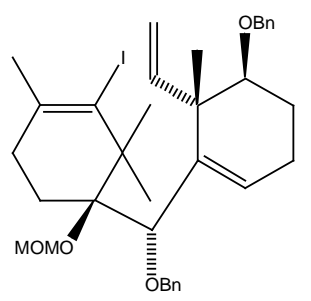

$[\alpha]_{D}^{27.2}+66.0$ (c 0.45, $\mathrm{CHCl}_{3}$ ); IR (thin film) $v_{\max }: 2948,1500,1152,1102,1030,918,734,698 \mathrm{~cm}^{-1}$; ${ }^{1} \mathrm{HNMR}\left(400 \mathrm{MHz}, \mathrm{CDCl}_{3}\right): \delta 7.31-7.21(\mathrm{~m}, 10 \mathrm{H}), 6.30(\mathrm{t}, J=6.9 \mathrm{~Hz}, 1 \mathrm{H}), 5.87(\mathrm{dd}, J=16.7,10.5 \mathrm{~Hz}$, $1 \mathrm{H}), 5.13(\mathrm{~d}, J=16.7 \mathrm{~Hz}, 1 \mathrm{H}), 5.13(\mathrm{~d}, J=10.5 \mathrm{~Hz}, 1 \mathrm{H}), 4.76(\mathrm{~d}, J=6.8 \mathrm{~Hz}, 1 \mathrm{H}), 4.75(\mathrm{~d}, J=6.8 \mathrm{~Hz}, 1 \mathrm{H})$, $4.64(\mathrm{~d}, J=11.0 \mathrm{~Hz}, 1 \mathrm{H}), 4.62(\mathrm{~d}, J=11.5 \mathrm{~Hz}, 1 \mathrm{H}), 4.45(\mathrm{~d}, J=11.5 \mathrm{~Hz}, 1 \mathrm{H}), 4.19$ (d, $J=11.0 \mathrm{~Hz}, 1 \mathrm{H})$, 4.03 (s, 1H), 3.41 (dd, $J=7.8,3.0 \mathrm{~Hz}, 1 \mathrm{H}), 3.30$ (s, 3H), 2.55 (ddd, $J=17.8,10.6,7.1 \mathrm{~Hz}, 1 \mathrm{H}), 2.41-2.32$ (m, 1H), 2.25-2.16 (m, 1H), 2.04-1.95 (m, 2H), 1.88-1.79 (m, 2H), $1.86(\mathrm{~s}, 3 \mathrm{H}), 1.62$ (ddd, $J=10.6,7.6$, $4.7 \mathrm{~Hz}, 1 \mathrm{H}), 1.46$ (s, 3H), 1.40 (s, 3H), 1.19 (s, 3H); ${ }^{13} \mathrm{C} \mathrm{NMR}\left(100 \mathrm{MHz}, \mathrm{CDCl}_{3}\right): \delta 146.6,139.1,139.0$, 
$138.1,136.3,129.8,128.1,127.9,127.5,127.2,127.1,126.8,119.5,113.9,92.5,83.3,83.1,80.6,71.2,69.4$, $55.5,48.5,46.0,31.4,30.1,30.0,27.9,26.6,23.4,21.5,19.3$; HRMS (FAB) $[\mathrm{M}+\mathrm{Na}]^{+}$calcd for $\mathrm{C}_{35} \mathrm{H}_{45} \mathrm{IO}_{4} \mathrm{Na}, 679.2260$, found, 679.2249; $\mathrm{R}_{\mathrm{f}}=0.46$ (hexane/ $\mathrm{AcOEt}=4 / 1$ ).

\section{$(1 S, 2 S, 7 S, 8 S)-2,7-B i s-b e n z y l o x y-1-m e t h o x y m e t h o x y-8,12,15,15$-tetramethyl-} tricyclo[9.3.1.03,8]pentadeca-3,11-diene (18)

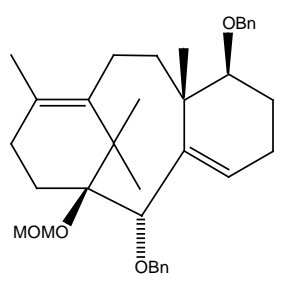

Vinyl iodide $17\left(0.0295 \mathrm{~g}, 4.50 \times 10^{-2} \mathrm{mmol}\right)$ was treated with a solution of $0.5 \mathrm{~N}$ 9-BBN in THF $(0.27 \mathrm{ml}$, $1.35 \times 10^{-1} \mathrm{mmol}$ ) at $0^{\circ} \mathrm{C}$, and the resulting mixture was refluxed for $3 \mathrm{~h}$ under an Ar atmosphere. After cooling to room temperature, to this mixture was added $\mathrm{H}_{2} \mathrm{O}(0.45 \mathrm{ml})$ and the solution was stirred for 15 min. This solution was added to a solution of $\mathrm{Pd}\left(\mathrm{PPh}_{3}\right)_{4}\left(0.0260 \mathrm{~g}, 2.25 \times 10^{-2} \mathrm{mmol}\right)$ in a mixture of $3 \mathrm{~N}$ $\mathrm{NaOH}\left(0.06 \mathrm{ml}, 1.80 \times 10^{-1} \mathrm{mmol}\right)$ and $\mathrm{MeCN}(4.5 \mathrm{ml})$, and the resulting mixture was refluxed for $72 \mathrm{~h}$ under an $\mathrm{Ar}$ atmosphere. The reaction was quenched with a mixture of $3 \mathrm{~N} \mathrm{NaOH}(0.270 \mathrm{ml})$ and $30 \% \mathrm{H}_{2} \mathrm{O}_{2}$ aqueous solution $(0.135 \mathrm{ml})$ for $2 \mathrm{~h}$ at $0{ }^{\circ} \mathrm{C}$. Then, the reaction mixture was diluted with $\mathrm{H}_{2} \mathrm{O}(10 \mathrm{ml})$ and extracted with $\mathrm{Et}_{2} \mathrm{O}(20 \mathrm{ml} \times 3)$. The combined organic layer was washed with brine $(20 \mathrm{ml})$, and concentrated under reduced pressure. The residue was purified by flash chromatography $\left(\mathrm{SiO}_{2}\right.$, hexane/ AcOEt (50:1)) to afford $\mathbf{1 8}(0.0202 \mathrm{~g}, 85 \%)$ as a viscous oil.

$[\alpha]^{21.8}+99.3\left(\mathrm{c} 0.65, \mathrm{CHCl}_{3}\right)$; IR (thin film) $v_{\max }: 2928,1454,1156,1104,1066,1026,918,788,754$ $\mathrm{cm}^{-1} ;{ }^{1} \mathrm{H}$ NMR $\left(400 \mathrm{MHz}, \mathrm{CDCl}_{3}\right) \delta$ 7.40-7.23 (m, 10H), 5.77(dd, $\left.J=4.9,2.4 \mathrm{~Hz}, 1 \mathrm{H}\right), 5.14(\mathrm{~d}, J=7.7 \mathrm{~Hz}$, $1 \mathrm{H}), 4.74(\mathrm{~d}, J=11.8 \mathrm{~Hz}, 1 \mathrm{H}), 4.50(\mathrm{~d}, J=7.7 \mathrm{~Hz}, 1 \mathrm{H}), 4.49(\mathrm{~d}, J=11.8 \mathrm{~Hz}, 1 \mathrm{H}), 4.45(\mathrm{~d}, J=11.0 \mathrm{~Hz}, 1 \mathrm{H})$, $4.14(\mathrm{~d}, J=11.0 \mathrm{~Hz}, 1 \mathrm{H}), 3.79(\mathrm{~s}, 1 \mathrm{H}), 3.73(\mathrm{dd}, J=12.0,3.8 \mathrm{~Hz}, 1 \mathrm{H}), 3.36(\mathrm{~s}, 3 \mathrm{H}), 2.37-2.13(\mathrm{~m}, 5 \mathrm{H})$, 2.89-1.93 (m, 4H), 1.76-1.58 (m, 3H), 1.40 (s, 3H), 1.33 (s, 3H), 1.05 (s, 3H), 1.02 (s, 3H); ${ }^{13} \mathrm{C}$ NMR (100 $\left.\mathrm{MHz}, \mathrm{CDCl}_{3}\right): \delta 143.7,139.4,139.1,134.3,131.1,128.3,128.1,127.9,127.5,127.2,127.0,118.5,93.5$, 87.0, 81.0, 75.8, 70.6, 69.7, 55.3, 42.0, 41.9, 33.3, 30.3, 28.2, 24.3, 24.2, 23.9, 22.3, 21.5, 20.9, 20.0; HRMS (FAB) $[\mathrm{M}]^{+}$calcd for $\mathrm{C}_{35} \mathrm{H}_{46} \mathrm{O}_{4}, 530.3396$, found, 530.3390; $\mathrm{R}_{\mathrm{f}}=0.55$ (hexane/ AcOEt $=4 / 1$ ). 